\title{
Magnetization reversal through synchronization with a microwave
}

\author{
Z. Z. Sun and X. R. Wang \\ Physics Department, The Hong Kong University of Science and Technology, Clear Water Bay, Hong Kong SAR, China \\ (Received 23 August 2006; published 2 October 2006)
}

\begin{abstract}
Based on the Landau-Lifshitz-Gilbert equation, it can be shown that a circularly polarized microwave can reverse the magnetization of a Stoner particle through synchronization. In comparison with magnetization reversal induced by a static magnetic field, it can be shown that when a proper microwave frequency is used the minimal switching field is much smaller than that of precessional magnetization reversal. A microwave needs only to overcome the energy dissipation of a Stoner particle in order to reverse magnetization, unlike the conventional method with a static magnetic field where the switching field must be of the order of magnetic anisotropy.
\end{abstract}

DOI: 10.1103/PhysRevB.74.132401

PACS number(s): 75.60.Jk, 05.45.Xt, 84.40.-x

\section{INTRODUCTION}

Magnetization reversal of single-domain magnetic nanoparticles (Stoner particles) ${ }^{1}$ is of significant interest in magnetic data storage and spintronics. Finding an effective way to switch magnetization from one state to another depends on our basic understanding of magnetization dynamics. Magnetization can be manipulated by laser, ${ }^{2}$ a polarized electric current, ${ }^{3,4}$ or a magnetic field. ${ }^{5}$ An important issue in magnetization reversal is the minimal switching field. Magnetization reversal using a static magnetic field ${ }^{5-8}$ or polarized electric current ${ }^{3,4}$ has received close attention in recent years, but there has been little investigation on microwave-induced magnetization reversal. Thirion et al. ${ }^{9}$ made probably the first attempt in this direction. It was shown that a dramatic reduction of the minimal switching field is possible by applying a small radio-frequency (rf) field pulse (the decrease in the static field is much larger than the amplitude of the rf field). Some numerical investigations in this direction were also given in Ref. 10. In this paper, it is shown that a circularly polarized microwave on its own can induce magnetization reversal. The minimal switching field depends on the microwave frequency. It can be shown that the minimal switching field is at a minimum at an optimal frequency. This optimal frequency is near the natural precession frequency at which the particle experiences the largest dissipation. At this optimal frequency, the switching field strength can be much smaller than the so-called Stoner-Wohlfarth (SW) limit ${ }^{11}$ and precessional magnetization switching field ${ }^{5-7}$ for a static magnetic field. Far from the optimal frequency, the switching field can be larger than the SW limit.

The minimal switching field was first studied by Stoner and Wohlfarth. ${ }^{11}$ The SW limit is the field at which the energy minimum around the initial state is destroyed and the target state is the only minimum, ${ }^{5-8}$ as illustrated in Figs. 1(a) and 1(b). In the absence of magnetic fields, two energy minima [ $A$ and $B$ in Fig. 1(a)], separated by a potential barrier $\Delta E$, are along the easy axis of a magnetic particle. At the SW limit, the original minimum near the initial state $A$ disappears [Fig. 1(b)], and the particle will end up at its unique minimum near the target state $B$. Recent theoretical and experimental studies ${ }^{5-7}$ have shown that the minimal switching field could be smaller than the SW limit. The reason has been explained earlier. ${ }^{8}$ As illustrated in Fig. 1(c), magnetization reversal can occur even when the minimum around $A$ exists. The reversal can happen as long as the particle energy at $A$ is higher than that at the saddle point $S P$, and the particle can pass through $S P$ under its own dynamics. It can be shown that the minimal switching field is of the order of the potential barrier $\Delta E .^{8}$

\section{FUNDAMENTAL DIFFERENCES BETWEEN TIME-INDEPENDENT AND TIME-DEPENDENT FIELDS}

The microwave-induced magnetization reversal is fundamentally different from that of a static magnetic field, because a static field is not an energy source while a microwave can be. This can be seen from the dynamic equation governing the evolution of a single-domain magnetic nanoparticle. For a particle with a magnetization of $\vec{M}=\vec{m} M_{s}, \vec{m}$ satisfies the Landau-Lifshitz-Gilbert (LLG) equation ${ }^{8,12}$

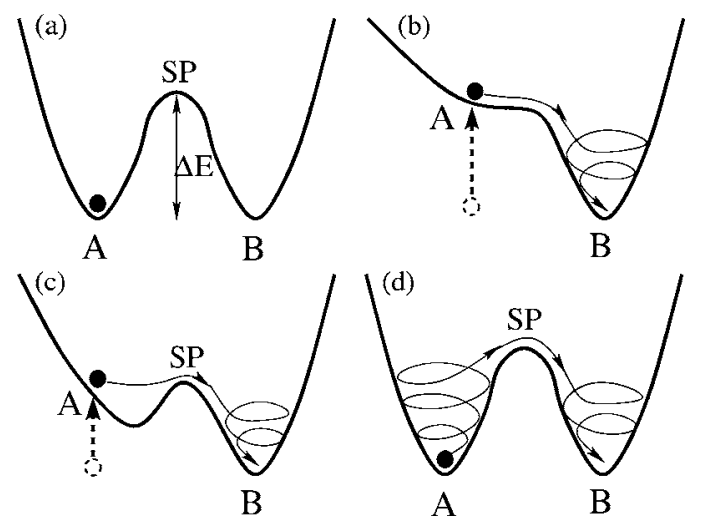

FIG. 1. Energy surface of a uniaxial magnetic particle in various schemes. $S P$ denotes the saddle point between two minima. (a) In the absence of magnetic fields: $A$ and $B$ are the two minima, separated by a potential barrier $\Delta E$. (b) At the SW limit: Target state $B$ is the only minimum. (c) Precessional magnetization reversal: The particle energy at $A$ is higher than that at $S P$ so that it can pass through $S P$ under its own dynamics. (d) New strategy: The system synchronizes its motion with a microwave, and climbs over the potential barrier to reverse its magnetization. 


$$
\left(1+\alpha^{2}\right) \frac{d \vec{m}}{d t}=-\vec{m} \times \vec{h}_{t}-\alpha \vec{m} \times\left(\vec{m} \times \vec{h}_{t}\right),
$$

where $M_{s}$ is the saturated magnetization of the particle, and $\alpha$ is a dimensionless damping constant. The total field, measured in unit of $M_{s}$, comes from an applied magnetic field $\vec{h}$ and the internal effective field $\vec{h}_{i}$ due to the magnetic anisotropy $w(\vec{m}, \vec{h}), \vec{h}_{t}=-\nabla_{\vec{m}} w(\vec{m}, \vec{h})=\vec{h}_{i}+\vec{h}$. In Eq. (1), time $t$ is in unit of $\left(|\gamma| M_{s}\right)^{-1}$ with $|\gamma|=2.21 \times 10^{5}(\mathrm{rad} / \mathrm{s}) /(\mathrm{A} / \mathrm{m})$ being the gyromagnetic ratio. From Eq. (1), the energy change rate for the particle can be obtained, ${ }^{13}$

$$
\frac{d w}{d t}=-\vec{m} \cdot \dot{\vec{h}}-\frac{\alpha}{1+\alpha^{2}}\left|\vec{m} \times \vec{h}_{t}\right|^{2}
$$

The second term due to the damping is always negative, while the first term due to the external magnetic field can be either positive or negative if the field varies with time. Thus, a time-dependent magnetic field can be both an energy source and energy sink.

\section{NEW STRATEGY}

Having explained that a microwave can be an energy source, the synchronization phenomenon of nonlinear dynamic systems ${ }^{14}$ can be used to reverse the magnetization of a Stoner particle by shining the particle with only a circularly polarized microwave. If the propagating direction of the microwave is along the particle's easy axis (the magnetic field rotates around the easy axis with the microwave frequency), the particle's magnetization in a synchronized motion precesses around the axis with the microwave frequency. As illustrated in Fig. 1(d), the magnetization starting from its initial minimum $A$ obtains energy from the microwave and eventually reaches its synchronized state. If the synchronized state is over the saddle point $S P$ and on the side of minimum $B$, the magnetization reversal is realized when the microwave radiation is turned off because magnetization will end up at minimum $B$ through the usual ringing effect. ${ }^{5}$ It is known that a nonlinear dynamic system under an external periodic field may undergo a nonperiodic motion other than synchronization. ${ }^{14}$ In general, the reversal criterion is: The magnetization is reversed if the system can cross the saddle point $S P$ in Fig. 1.

To demonstrate the feasibility of the new strategy, a uniaxial magnetic anisotropy is considered,

$$
w(\vec{m}, \vec{h}=0)=-k m_{x}^{2},
$$

where $k>0$ measures the anisotropy strength. Without losing the generality, $k$ [used as a scale for the field strength according to Eq. (1)] shall be set to 1 . The easy axis is chosen to be along the $x$ axis rather than the $z$ axis because the north and south poles are singular in spherical coordinates, and it is more convenient to locate the minima $A$ and $B$ (Fig. 1) away from the singularities.

\section{MULTIPLE SYNCHRONIZATION SOLUTIONS}

Under a circularly polarized microwave of amplitude $h_{0}$ and frequency $\omega$,

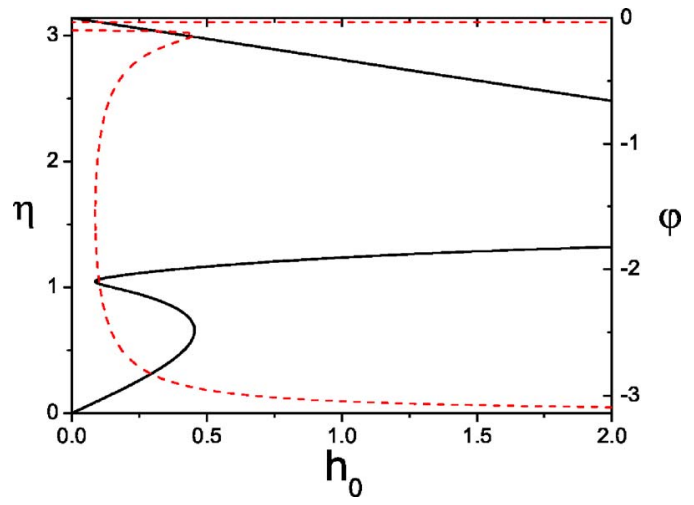

FIG. 2. (Color online) Graphic demonstration of multiple synchronization solutions. The solid lines are from Eq. (6) and the dashed lines are from Eq. (7). The graph is plotted at $\alpha=0.1$ and $\omega=1$.

$$
\vec{h}(t)=h_{0}[\cos (\omega t) \hat{y}+\sin (\omega t) \hat{z}],
$$

and the synchronized motion is

$$
\vec{m}(t)=\cos \eta \hat{x}+\sin \eta[\cos (\omega t+\varphi) \hat{y}+\sin (\omega t+\varphi) \hat{z}]
$$

where $\eta$ (a constant of motion) is the precessional angle between $\vec{m}$ and the $x$ axis. $\varphi$ is the locking phase in the synchronized motion. By substituting Eqs. (3)-(5) into Eq. (1), $\eta$ and $\varphi$ satisfy

$$
\begin{gathered}
\sin \eta \sqrt{\alpha^{2} \omega^{2}+(2-\omega / \cos \eta)^{2}}=h_{0}, \\
\sin \varphi=-\alpha \omega \sin \eta / h_{0},
\end{gathered}
$$

where $\eta \in[0, \pi]$. For fixed $\left(h_{0}, \omega, \alpha\right), \eta$ and $\varphi$ may have multiple solutions. As illustrated in Fig. 2, the solutions of $\eta$ (solid lines) are plotted as a function of $h_{0}$ for $\omega=1$ and $\alpha$ $=0.1$. The dashed lines denote the corresponding $\varphi$. Multiple solutions of $\eta, \varphi$ are evident. For example, there are four solutions of $\eta$ when $h_{0} \in[0.09,0.45]$. Numerically, it can be shown that two solutions at around $\eta=1$ (in between) are unstable, while the other two near $\eta=0, \pi$ are stable. Thus, the system shall eventually end up at one of the two stable solutions. Which one the system will choose depends on the initial condition. For a given initial condition $[\vec{m}(0)=\hat{x}$ in this study], the system picks the solution near $\eta=\pi$ when $h_{0}$ is larger than a critical value called the minimal switching field. According to our reversal criterion, the magnetization is reversed through synchronization.

\section{NUMERICAL VERIFICATION OF SYNCHRONIZED AND NONSYNCHRONIZED MOTION}

A nonlinear dynamic system under an external periodic field may undergo motion other than synchronized. Unfortunately, a nonsynchronized motion is, in general, hard to define analytically. Usually, reliance must be placed on the numerical method. In terms of the LLG equation under a circularly polarized microwave of Eq. (4), it is straightforward ${ }^{8}$ to calculate numerically $\vec{m}(t)$ starting from $\vec{m}(0)=\hat{x}$. The upper inset of Fig. 3 is the trajectory of $\vec{m}(t)$ 


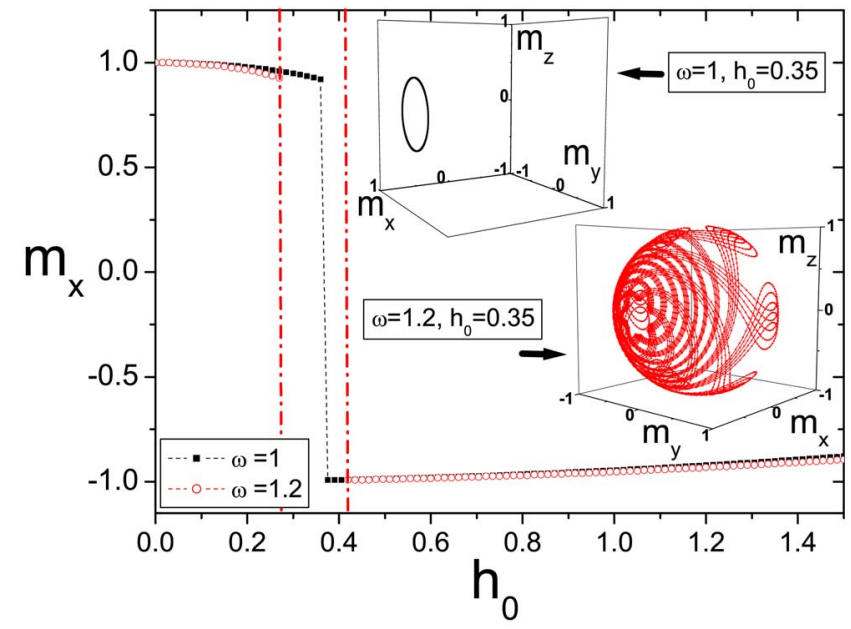

FIG. 3. (Color online) $m_{x}$ of synchronized motion vs $h_{0}$ for $\alpha$ $=0.1$ and $\omega=1$ (filled squares); 1.2 (open circles). Nonsynchronized motion when $h_{0} \in[0.27,0.42]$ (between two dash-dotted lines) is found for $\omega=1.2$. Upper inset: Long time trajectory of $\vec{m}(t)$ for $\omega$ $=1$ and $h_{0}=0.35$. Lower inset: Long time trajectory of $\vec{m}(t)$ for $\omega$ $=1.2$ and $h_{0}=0.35$.

after long time in $m_{x} m_{y} m_{z}$ space for $h_{0}=0.35, \omega=1$, and $\alpha$ $=0.1$. A simple closed loop in a plane parallel to the $y z$ plane indicates that this is a synchronized motion. Alternatively, the lower right inset of Fig. 3 is the long time trajectory of $\vec{m}(t)$ for $h_{0}=0.35, \omega=1.2$, and $\alpha=0.1$. Its motion is very complicated, corresponding to a nonsynchronized motion. It is found that whether the motion is synchronized or not is sensitive to the microwave frequency. For example, all motions for $\omega=1$ are synchronized, while both synchronized and nonsynchronized motions are possible for $\omega=1.2$. The motion is nonsynchronized for $h_{0}$ in the range of [0.27, 0.42], while it is synchronized for other values of $h_{0}$. Figure 3 is $m_{x}$ of synchronized motions as a function of $h_{0}$ for $\omega=1$ and 1.2.

\section{OPTIMAL MICROWAVE FREQUENCY}

Using the reversal criterion given earlier, it can be shown from Fig. 3 that the minimal switching field $h_{c}$ is about 0.375 for $\omega=1$ because $m_{x}$ in the synchronized motion is negative when $h_{0}>h_{c}$. For $\omega=1.2$, the minimal switching field takes a value at which the magnetization undergoes a nonsynchronized motion. Numerically, it can be shown that $\vec{m}$ crosses the $y z$ plane when $h_{0} \geq 0.285$. Thus, the minimal switching field is determined as $h_{c}=0.285$ for $\omega=1.2$. The reason that the value of the minimal switching field is so sensitive to the microwave frequency is because a switching field, as illustrated in Fig. 1(d), needs to overcome the dissipation which is related to the motion of the magnetization (see the LLG equation). To reveal the frequency dependence of the minimal switching field, Fig. 4 shows the minimal switching field $h_{c}$ vs the microwave frequency $\omega$ for various $\alpha=0,0.001$, $0.1,1$, and 1.5. $\omega=0$ corresponds to the case of a static field along the $y$ axis. The curve of $\alpha=0$ intersects the $h_{c}$ axis at $h_{c}=1$, which agrees with the exact minimal switching field $h_{c}=1 .^{8}$ The intersections of all other curves of $\alpha \neq 0$ are the

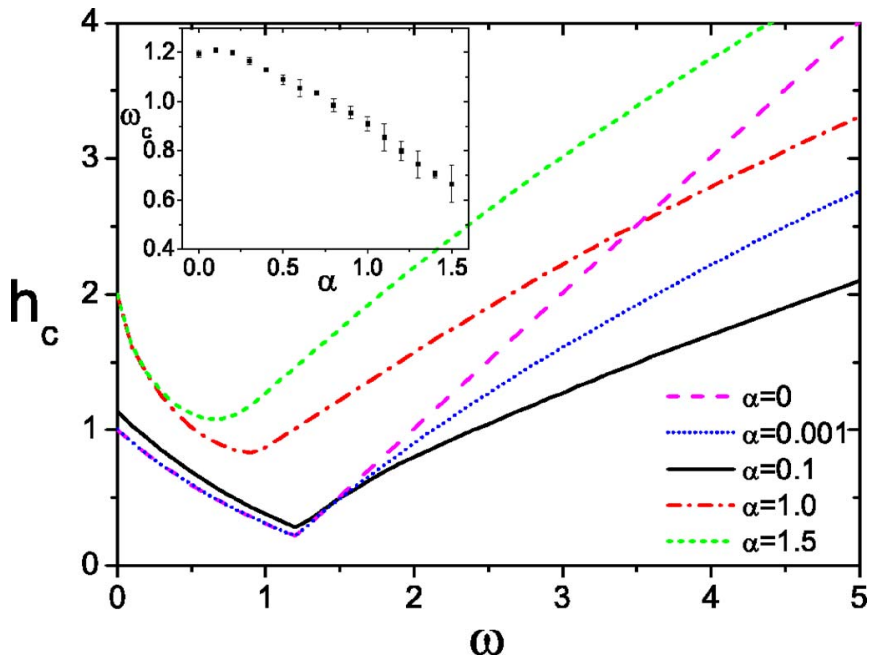

FIG. 4. (Color online) The minimal switching field $h_{c}$ vs $\omega$ for various damping constants $\alpha=0,0.001,0.1,1$, and 1.5. Inset: The optimal frequency $\omega_{c}$ vs $\alpha$.

same as those with a static field. ${ }^{5,8}$ When $\alpha \geq 1$, it becomes the SW limit $h_{c}=2$. For a given $\alpha$, Fig. 4 shows the existence of an optimal microwave frequency, $\omega_{c}$, at which the minimal switching field is the smallest. Far from the optimal frequency, the minimal switching field can be larger than the SW limit. The inset of Fig. 4 is $\omega_{c}$ vs $\alpha$. The optimal frequency is near the natural precessional frequency at which the dissipation is a maximum.

\section{SWITCHING FIELD AS A FUNCTION OF DISSIPATION}

From the above discussions, it can be seen that the minimal switching field is a minimum at the optimal frequency $\omega_{c}$. The square symbols in Fig. 5 are the minimal switching

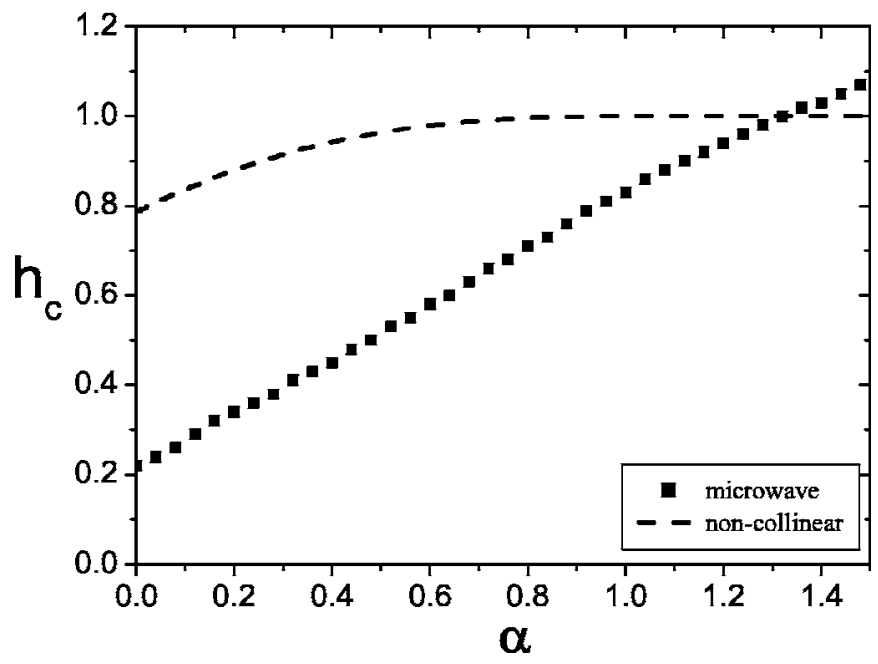

FIG. 5. $h_{c}$ vs $\alpha$ for the uniaxial model of Eq. (3) under different reversal schemes. Square symbols are the numerical results of $h_{c}$ at the optimal frequency in the present strategy with a circularly polarized microwave. The dashed line is $h_{c}$ under a noncollinear static field of $135^{\circ}$ to the easy axis. It saturates to the SW limit beyond $\alpha=1$ (Ref. 8). 
fields at $\omega_{c}$ with different damping constant $\alpha$ for the uniaxial model of Eq. (3). They follow approximately the line of $h_{c} \approx 0.23+0.58 \alpha$. This approximate linear relation is related to the fact that the damping (field) is proportional to $\alpha$. For comparisons, the minimal switching fields of a precessional magnetization reversal under a static magnetic field, which saturates to the SW limit beyond $\alpha=1$, is also plotted in Fig. 5. It can be seen that for small damping, the smallest (at the optimal frequency) minimal switching field can be much smaller than that in the precessional magnetization reversal. For large damping, the switching field can be larger than the SW limit.

\section{DISCUSSION AND CONCLUSIONS}

It is important to compare the present strategy with other strategies involving time-dependent fields. Firstly, the current scheme is fundamentally different from that in the experiment of Thirion et al. ${ }^{9}$ in several aspects. (i) A circularly polarized microwave of fixed frequencies is the only switching field in the new scheme, while in Ref. 9, a linear polarized $\mathrm{rf}$ field is used as an additional external field to reduce the main static switching magnetic field. (ii) For a Co particle of $H_{i}=10^{5} \mathrm{~A} / \mathrm{m},{ }^{6}$ the optimal frequency is about order of $10 \mathrm{GHz}$ rather than order of $1 \mathrm{GHz}$ employed in Ref. 9. At a few GHz, Fig. 4 shows that the switching field would be too large to have any advantage over a static field. The current scheme is also very different from that in Ref. 13 in many aspects. (i) The time-dependent field in Ref. 13 is used as a ratchet that should be adjusted with the motion of magnetization. In contrast, the present scheme is based on the synchronization phenomenon in nonlinear dynamics such that a circularly polarized microwave of fixed frequencies is used and the magnetization motion is synchronized with the microwave in the reversal process. (ii) The switching field in Ref. 13 is in general nonmonochromatic and very complicated, requiring a precise control of time-dependent polarization. Thus, it would be a great challenge to generate such a field. Alternatively, the current scheme is much easier to implement and it could be technologically important.

In conclusion, a circularly polarized, microwave-induced magnetization reversal is proposed. The proposal is based on the facts that a microwave can constantly supply energy to a Stoner particle, and the magnetization motion can be synchronized with the microwave. It can be demonstrated that a Stoner particle under the radiation of a circularly polarized microwave can indeed move out of its initial minimum and climb over the potential barrier. The switching field at the optimal microwave frequency will be much smaller than the SW limit and that of the precessional magnetization reversal for small damping.

\section{ACKNOWLEDGMENT}

This work is supported by UGC, Hong Kong, through RGC CERG grants (No. 603106).
${ }^{1}$ Spin Dynamics in Confined Magnetic Structures I \& II, edited by B. Hillebrands and K. Ounadjela (Springer-Verlag, Berlin, 2001).

${ }^{2}$ M. Vomir, L. H. F. Andrade, L. Guidoni, E. Beaurepaire, and J.-Y. Bigot, Phys. Rev. Lett. 94, 237601 (2005).

${ }^{3}$ J. Slonczewski, J. Magn. Magn. Mater. 159, L1 (1996); L. Berger, Phys. Rev. B 54, 9353 (1996).

${ }^{4}$ M. Tsoi, A. G. M. Jansen, J. Bass, W. C. Chiang, M. Seck, V. Tsoi, and P. Wyder, Phys. Rev. Lett. 80, 4281 (1998); E. B. Myers, D. C. Ralph, J. A. Katine, R. N. Louie, and R. A. Buhrman, Science 285, 867 (1999); J. A. Katine, F. J. Albert, R. A. Buhrman, E. B. Myers, and D. C. Ralph, Phys. Rev. Lett. 84, 3149 (2000); J. Z. Sun, Phys. Rev. B 62, 570 (2000); Z. Li and S. Zhang, ibid. 68, 024404 (2003); Y. B. Bazaliy, B. A. Jones, and S. C. Zhang, ibid. 57, R3213 (1998); 69, 094421 (2004).

${ }^{5}$ L. He, W. D. Doyle, and H. Fujiwara, IEEE Trans. Magn. 30, 4086 (1994); L. He and W. D. Doyle, J. Appl. Phys. 79, 6489 (1996).

${ }^{6}$ W. K. Hiebert, A. Stankiewicz, and M. R. Freeman, Phys. Rev. Lett. 79, 1134 (1997); C. H. Back, D. Weller, J. Heidmann, D. Mauri, D. Guarisco, E. L. Garwin, and H. C. Siegmann, ibid. 81, 3251 (1998); C. H. Back, R. Allenspach, W. Weber, S. S. P. Parkin, D. Weller, E. L. Garwin, and H. C. Siegmann, Science 285, 864 (1999); Y. Acremann, C. H. Back, M. Buess, O. Port- mann, A. Vaterlaus, D. Pescia, and H. Melchior, ibid. 290, 492 (2000).

${ }^{7}$ H. W. Schumacher, C. Chappert, P. Crozat, R. C. Sousa, P. P. Freitas, J. Miltat, J. Fassbender, and B. Hillebrands, Phys. Rev. Lett. 90, 017201 (2003); H. W. Schumacher, C. Chappert, R. C. Sousa, P. P. Freitas, and J. Miltat, ibid. 90, 017204 (2003); T. M. Crawford, T. J. Silva, C. W. Teplin, and C. T. Rogers, Appl. Phys. Lett. 74, 3386 (1999); M. Bauer, J. Fassbender, B. Hillebrands, and R. L. Stamps, Phys. Rev. B 61, 3410 (2000).

${ }^{8}$ Z. Z. Sun and X. R. Wang, Phys. Rev. B 71, 174430 (2005).

${ }^{9}$ C. Thirion, W. Wernsdorfer, and D. Mailly, Nat. Mater. 2, 524 (2003).

${ }^{10}$ K. Rivkin and J. B. Ketterson, cond-mat/0511497 (unpublished).

${ }^{11}$ E. C. Stoner and E. P. Wohlfarth, Philos. Trans. R. Soc. London, Ser. A 240, 599 (1948).

${ }^{12}$ L. Landau and E. Lifshitz, Phys. Z. Sowjetunion 8, 153 (1935); T. L. Gilbert, Phys. Rev. 100, 1243 (1955).

${ }^{13}$ Z. Z. Sun and X. R. Wang, Phys. Rev. B 73, 092416 (2006); Phys. Rev. Lett. 97, 077205 (2006).

${ }^{14}$ Z. Z. Sun, H. T. He, J. N. Wang, S. D. Wang, and X. R. Wang, Phys. Rev. B 69, 045315 (2004); X. R. Wang, J. N. Wang, B. Q. Sun, and D. S. Jiang, ibid. 61, 7261 (2000); X. R. Wang and Q. Niu, ibid. 59, R12755 (1999). 